\title{
Carnets
}

Revue électronique d'études françaises de l'APEF

Première Série - 1 Numéro Spécial | 2009

Cultures littéraires : nouvelles performances \& développement

\section{Enjeux et limites de la création lectorale}

\section{Alain Trouvé}

\section{OpenEdition}

1 Journals

Édition électronique

URL : http://journals.openedition.org/carnets/3970

DOI : $10.4000 /$ carnets.3970

ISSN : 1646-7698

Éditeur

APEF

Édition imprimée

Date de publication : 1 juin 2009

Pagination : 271-280

Référence électronique

Alain Trouvé, "Enjeux et limites de la création lectorale », Carnets [En ligne], Première Série - 1 Numéro Spécial | 2009, mis en ligne le 16 juin 2018, consulté le 03 mai 2019. URL : http://

journals.openedition.org/carnets/3970; DOI : 10.4000/carnets.3970

\section{(c) (i) (9)}

Carnets est mis à disposition selon les termes de la licence Creative Commons - Atribution - Pas d'utilisation commerciale 4.0 International. 


\title{
ENJEUX ET LIMITES DE LA CRÉATION LECTORALE
}

ALAIN TROUVÉ

Université de Reims

alain.trouve@wanadoo.fr

\begin{abstract}
Résumé
II s'agit de montrer à quelles conditions le lecteur peut, lisant un texte d'écrivain, se faire en quelque sorte inventeur d'un sens nouveau échappant à l'intention de ce dernier. C'est l'implication de l'écrivain comme sujet total, conscient et inconscient mêlés, qui signe la syncope du sens à l'origine de tout acte créateur. La lecture créatrice se fonde sur ce "point aveugle». Elle passe par une performance verbale dont le texte lu est le référent. L'" effet littérature » résulte du croisement des performances auctoriale et lectorale. Pour autant les droits du lecteur ne sont pas illimités. Violer la lettre du texte d'auteur pour lui en subsister un autre en tordant pour l'occasion la théorie du texte fantôme, c'est tout simplement gommer la spécificité du littéraire, fondée sur le décalage entre un sens immédiatement perceptible et d'autres couches voilées ou potentielles, et ruiner la possibilité d'une interprétation créatrice. Remettre en perspective les rôles de l'auteur et du lecteur n'est pas nier la créativité de ce dernier, c'est au contraire lui permettre de donner toute sa mesure.
\end{abstract}

\section{Abstract}

This paper aims at showing under what conditions the reader can, in reading a writer's text, invent a new meaning which escapes the latter's intention. It is the writer's implication as total subject, intertwined conscious and subconscious, which produces the "syncope of meaning" leading to any authentic creative act. Violating the letter of an author's text to impose another by twisting the theory of a ghost text for the occasion simply amounts to erasing the specificity of the literary text, which lies in the gap between immediately visible meaning and other potential or hidden strata of meaning, and to ruining the possibility of creative interpretation. Putting the roles of author and reader back into perspective does not mean negating the latter's creativity ; on the contrary, it amounts to giving him the opportunity to show the extent of his talent.

Mots-clés: Lecture, Interprétation, Inconscient, Création, Texte de lecture

Keywords: Reading, Meaning, Subconscious, Creative act, Counter-text from reading 
Depuis l'avènement des théories de la lecture, dans le dernier quart du vingtième siècle, il est devenu presque banal d'envisager le littéraire comme le fruit d'une coopération entre auteur et lecteur, voire comme une création partagée. Mais ce quasi lieu commun recouvre bien des malentendus, des confusions entre interprétation du geste créateur, donc reproduction d'un sens déjà là, et authentique production de circuits inédits de la signification.

Cette communication se propose de montrer à quelles conditions le lecteur peut, lisant un texte d'écrivain, se faire en quelque sorte inventeur d'un sens nouveau échappant à l'intention de ce dernier.

Le point de vue que j'adopte est celui d'une théorie de la lecture s'inscrivant dans une théorie du littéraire. En toile de fond: la littérature comme pratique esthétique historiquement datée (Rancière, 2007), fondée depuis la Révolution sur un partage des rôles entre l'auteur et son lectorat. A l'auteur, l'impulsion créatrice, à son lecteur la sanction de la valeur esthétique et l'appropriation du texte impliquant une marge de création interprétative.

On percevra dans mon propos une distance vis-à-vis d'un certain nivellement des productions langagières, déduites d'une application radicale des théories de l'hypertextualité et de la déconstruction. Peut-être aussi, dans une époque où il y a tant de candidats au statut d'auteur et si peu de lecteurs véritables, l'idée que la littérature au plein sens du terme ne peut vivre que de la collaboration entre les deux pôles de la relation littéraire.

En ce sens, j'examinerai les conditions de la création lectorale, puis ses limites, afin de mieux cerner la spécificité d'une lecture dite littéraire.

\section{Invention scripturale et création lectorale}

\section{La création auctoriale comme condition première}

Posons d'emblée l'axiome suivant: la lecture littéraire ne peut oublier l'artefact auctorial, elle prend son essor à partir de lui. En un sens, la création lectorale est d'abord recréation, reconnaissance de la qualité du texte d'auteur et cette dernière est déjà une affaire de lecture. Considérons par exemple Sanctuaire, d'abord écrit par Faulkner en 1929 dans l'intention de coller aux standards d'un genre à succès : intrigue policière, thèmes provocateurs du viol et du trafic d'alcool sur fond de prohibition. Entre la version initiale et ses réécritures, celle de 1931 en particulier, se déploient tout le dispositif d'invention faulknérien et notamment cette narration multifocale totalement décentrée, court-circuitant l'élaboration d'une construction rationalisée. Ainsi le stéréotype du roman policier est traité sous une forme déceptive et transgressive n'aboutissant pas à une élucidation des situations décrites dans la plus grande confusion : le viol supposé de Temple Drake et le meurtre de Tommy. Autour ou à partir de ces scènes, le texte de Faulkner donne à penser et à rêver sur 
le mal et la condition humaine, sur la barrière toute relative séparant normalité et anomalie, ordre moral et délinquance supposée. Dans le décor qui réunit aux premières pages Popeye et l'avocat Horace Benbow est évoqué un chant d'oiseau qui pourrait se lire comme l'emblème du roman faulknérien fondé sur la tension entre compréhension et obscurité.

Derrière lui, l'oiseau chanta de nouveau, trois mesures monotones, constamment répétées : un chant à la fois dépourvu de sens et profond, qui s'éleva du silence plein de soupirs et de paix dans lequel le lieu semblait s'isoler, et d'où surgit, l'instant d'après, le bruit d'une automobile qui passa dans le lointain et mourut dans le lointain. (Sanctuaire, p. 14)

Ce « lui » que le texte réfère de manière équivoque à chacun des deux personnages est applicable par extension au lecteur plongé dans le silence de sa lecture et confronté aux déflagrations nées de la scène romanesque.

En termes plus généraux: l'artefact auctorial se reconnaît comme création digne d'être explorée par le lecteur en raison de trois qualités au moins: surplus de sens/polysémie de l'énoncé (c'est la théorie qui naît avec les formalistes russes et fut reprise notamment par Barthes), rupture avec les codes dominants (Riffaterre avança à propos de la poésie son concept d'agrammaticalité), intrication de motions conscientes et inconscientes susceptible de générer la fonction réparatrice du jeu (fonction elle-même partagée entre les deux pôles du littéraire) : je fais ici allusion aux travaux de Winnicott sur l'art comme jeu (Jeu et réalité, 1971), propos développés naguère par Michel Picard (La lecture comme jeu, 1986) et repris plus récemment sous un angle différent par Jean-Marie Schaeffer (Pourquoi la fiction ?, 1999)

\section{Dimension herméneutique de la création lectorale}

Pour le dire autrement encore et avec Bertrand Gervais ( $A$ l'écoute de la lecture, 1993), le texte littéraire digne de ce nom offre prise à une double lecture, en progression et en compréhension, la seconde à dimension essentiellement interprétative correspondant à l'espace de la création lectorale. Qu'une partie du sens puisse échapper à son créateur avait été en son temps décrit par Umberto Eco sous la tripartition de l'intention signifiante : intentio scriptoris, intentio operis, intentio lectoris. Toutefois, il s'agissait surtout et avec raison dans Les limites de l'interprétation d'indiquer en quoi les productions de l'intentio lectoris sont illégitimes, non de comprendre en quoi le lecteur peut réellement innover. S’il paraît pertinent d'écarter les élucubrations posées en toute méconnaissance du texte prétendument interprété, le risque existe parallèlement, sous prétexte de préserver l'adéquation de la 
lecture à l'œuvre, de ne concevoir l'interprétation que comme la redite d'un sens préalablement posé qui enserrerait le lecteur dans les pas de l'auteur.

Quels sont donc les points aveugles de l'intentio auctoris susceptibles de servir de tremplin à la création lectorale au plein sens du terme? Deux directions peuvent être mentionnées.

L'une, qui insère l'auteur dans le collectif, renvoie à la fonction d'anticipation de l'œuvre d'art, porteuse de significations qui excèdent l'époque ou le moment. L'écriture littéraire qui joue avec les mythes en tant que produits de l'imaginaire collectif peut ainsi parfois contribuer à en forger de nouveaux sous l'impulsion d'un créateur identifié, comme l'a montré Jean Rousset à propos du Dom Juan de Tirso de Molina. L'écriture littéraire rencontre ici la notion d'inconscient collectif forgée en son temps par Jung et dont on sait que la pensée freudienne fit une critique sévère surtout pour sa prétention à se substituer à l'interprétation individualisée des mécanismes de refoulement. Mais on peut aussi concevoir cette notion en lui donnant son acception d'insu sans faire intervenir les mécanismes de refoulement au sens freudien. Certaines structures de pensée qui participent de l'élaboration des œuvres excèdent en effet la conscience qu'en prend l'auteur, on pense notamment aux structures de l'imaginaire telles qu'ont pu les décrire Bachelard ou Gilbert Durand, structures dont l'identification est susceptible d'enrichir l'interprétation. La remarque vaut sans doute autant pour les langues dont les réseaux souterrains sont alimentés au feu du désir croisé des utilisateurs : "Nuestras lenguas estan surcadas por la memoria y el deseo » / "Nos langues sont sillonnées par la mémoire et le désir » note ainsi le romancier Carlos Fuentes à la fin de son roman La dos orillas, Les deux rives (Fuentes, 2007, 176). Ce qui se dit ici à propos de l'échange entre le Nouveau Monde et l'Ancien vaut sans doute pour l'échange entre toutes les cultures.

L'autre direction est celle d'un inconscient individuel renvoyant à un parcours personnel. II a en ce sens été maintes fois remarqué que l'écriture relève d'une aventure du sens, autrement dit d'un jeu plus ou moins maîtrisé avec le matériau pulsionnel ${ }^{1}$. Cette aventure se concrétise notamment en scénario, images et réminiscences intertextuelles.

Scénario : derrière la façade rationalisée de la trame narrative s'effectue aussi un jeu avec les pulsions archaïques. Le roman puise en effet aux sources du roman familial et de ses deux structures fondamentales, décrites par Marthe Robert: structure œdipienne du Bâtard et structure préœdipienne de l'Enfant trouvé (Robert, 1972). Parce que le complexe d'CEdipe joue un rôle nucléaire dans l'accès au Symbolique, on peut supposer que la structure organisée de l'œuvre lui est liée ; de fait c'est cette structuration qui relève le plus

\footnotetext{
${ }^{1}$ On peut rappeler la fameuse formule de Jean Ricardou appliquée au Nouveau roman, formule préférant «l'aventure d'une écriture à l'écriture d'une aventure ». Ou citer la formule non moins célèbre appliquée par Rimbaud au Je poétique : « Je est un Autre».
} 
directement de l'élaboration consciente de l'auteur. En revanche, tout ce qui se rattache aux orientations préœdipiennes de la libido a partie liée avec le chaos et le désordre qui s'immisce au sein du geste créateur. L'ouverture aux motions inconscientes semble ainsi s'opérer chez de nombreux auteurs selon deux modalités fréquemment successives: modalité forte plaçant le chaos formel au premier plan des structures narratives, modalité tempérée reléguant le désordre au second plan dans le traitement poétique des images. On peut évoquer en ce sens Le Clézio (Le Procès-verbal, vs La Ronde, Révolution, Onitsha), Aragon (Les Aventures de Télémaque, La Défense de l'infini vs Aurélien, La Semaine Sainte) ; Vargas Llosa (Les chiots vs Tours et détours de la vilaine fille). Faulkner ou Joyce feraient de leur côté du chaos de la vision narrative un principe plus constant d'écriture, que régit en dernier ressort une cohérence poétique, toujours à reconstruire. On peut encore citer en ce sens les romans de la suissesse Catherine Colomb dont chaque phrase comme saturée de sens paraît s'ouvrir sur l'écheveau de la mémoire, romans qui comme Châteaux en enfance ou Les Esprits de la terre substituent à la construction classique d'un temps linéaire la cohérence d'un imaginaire nourri à ce que Pascal Quignard nomme le Jadis. Nul doute que ces écritures offrent au lecteur la possibilité de déployer davantage sa créativité.

La dernière source d'invention lectorale prend appui sur le repérage d'intertextes latents (Trouvé, 2007) susceptibles d'avoir échappé à la perception de l'auteur. Contrairement à son acception courante de continuité textuelle exogène, l'intertexte dans son acception latente renvoie à un texte refoulé pour des raisons qui appartiennent à l'économie de production de l'œuvre, à un moment où l'auteur se détourne ostensiblement d'un autre auteur ou d'une œuvre jadis prisée. Sur le modèle du déplacement au sens freudien et de l'intertexte pensé en termes d'isotopie (Arrivé, 1973), il se présente comme collection d'éléments disjoints relevant d'une même unité structurelle : le texte exogène masqué.

On entrevoit ainsi la marge d'invention proprement dite offerte au lecteur. Reste à en poser à présent les limites.

\section{Contre le nivellement des textes et l'abus de la confusion des genres}

S’il n'est de lecture dotée de quelque consistance que mise en mots, donc mise en texte - tel est ici le second axiome que je voudrais proposer -, il est tentant mais finalement peu efficace de fondre tous les textes dans un vaste ensemble au nom du principe d'engendrement universel.

La lecture littéraire telle que nous la concevons s'apparente à un commentaire créatif, non à une réécriture au plein sens du terme ; elle relève, pour parler en termes genettiens du 
métatexte et non de l'hypertexte. Elle se dissocie de ce que Eco dénonçait déjà comme lecture prétextuelle (Eco, 1992, 46).

Essayons d'indiquer par quel changement de perspective s'opère le glissement d'une catégorie à l'autre. L'hypertextualité renvoie certes à l'image de l'écrivain comme lecteur de ses confrères. Mais cette lecture s'effectue selon un changement de focale en vertu duquel le texte lu cesse d'être le foyer principal du sens.

Ainsi le Candide de Voltaire peut sans aucun doute être interprété comme une lecture critique de la philosophie optimiste de Leibniz et de son disciple Wolf. Les guerres auxquelles se trouve mêlé le héros, le tremblement de terre de Lisbonne, opposent le démenti du mal politique ou naturel à l'idée de l'Optimisme et du « meilleur des mondes possibles ". Mais ce niveau métaphysique de l'écriture se tresse à d'autres couches de sens qui en excèdent la portée. On peut aussi lire ce conte philosophique comme une critique sociale de la noblesse par le bourgeois François-Marie Arouet. Les figures du baron de Thunder-ten-Tronckh et de sa caricaturale famille campent plaisamment la morgue des seigneurs, vestiges d'une époque condamnée à brève échéance. L'anticléricalisme prend également un tour beaucoup plus accusé qui excède la place de la religion chez le philosophe allemand et renvoie à une Europe encore en proie à l'Inquisition.

Dans une nouvelle de son recueil Mille regrets (1942), Elsa Triolet poursuit un dialogue littéraire avec Albert Camus. À la philosophie de l'absurde, qui place la condition humaine sous la lumière de la mort inéluctable, égalisatrice de toutes choses, elle oppose dans Quel étranger qui n'est pas d'ici ou Le mythe de la baronne Mélanie I'histoire cocasse d'une femme revivant sa vie à l'envers et découvrant le caractère inoffensif de toute douleur, suivie de l'état de paix qui la précédait à «l'aller». Le «mythe burlesque de Mélanie d'Aubrey » opère donc une sorte de surenchère ironique sur la pensée de l'absurde dont l'auteur de L'Etranger reconnut l'efficacité : «Quant à votre point de départ, la critique de mon essai, vous avez tout à fait raison : il peut y avoir un mythe absurde, mais la pensée absurde n'est pas possible. C'est en effet une pensée du «retour » et non de l'aller » (Camus, 1943) ${ }^{2}$.

Pourtant cette nouvelle, tout comme le conte voltairien, ne se peut réduire à son hypotexte et vaut aussi par la nouveauté de la veine littéraire qu'elle manifeste, mêlant invention, comique et secrète exploration d'une pensée tournée vers cet autre impossible : l'origine.

Ces deux exemples d'une création ou recréation littéraire pour dessiner, donc, un champ qui n'est plus tout à fait celui d'une lecture littéraire, ce dont semblent ne pas s'aviser certains critiques et universitaires actuels séduits par la théorie de l'énoncé fantôme,

\footnotetext{
${ }^{2}$ Albert Camus, lettre à Elsa Triolet du 23 mai 1943, citée dans CEuvres Romanesques Croisées d'Aragon et d'Elsa Triolet, Paris, Robert Laffont, 1964-1974, 42 volumes, 3, p. 39.
} 
naguère avancée par Michel Charles. Or cette théorie, proposée dans l'Introduction à l'étude des textes (1995), s'applique peut-être plus judicieusement à l'interprétation, quoi qu'en ait son concepteur ${ }^{3}$, qu'à la production d'énoncés nouveaux: “On appellera éléments fantômes ces éléments d'un texte qui, tout en étant doués d'efficacité, demeurent en quelque sorte cachés pour laisser au texte sa lisibilité »(Charles, 1995).

La mise au jour d'énoncés fantômes peut en ce sens participer de la restitution d'un sens plus complexe que celui que privilégie le lissage final de l'écriture. Elle permet également de proposer d'intéressantes cohérences interprétatives dans des textes que dominent le chaos et l'écriture fragmentaire ${ }^{4}$. On ne saurait en revanche, à notre avis, ériger le texte possible écarté de la rédaction effective, en équivalent du texte à lire comme s'y emploient certains critiques en proie à ce que j'appellerais volontiers le complexe du critique romancier $^{5}$. Je prendrai pour exemple les réécritures auxquelles procède Pierre Bayard. Avec la virtuosité et l'humour qu'on lui connaît, son dernier essai entreprend de démontrer l'erreur commise par Sherlock Holmes et par son auteur, Conan Doyle, dans son célèbre récit Le Chien des Baskerville. La réécriture ludique l'emporte dans L'Affaire du chien des Baskerville (Bayard, 2008) sur l'analyse littéraire, mais une telle démarche, quels que soient les charmes de l'hypertexte ainsi produit, tend à ruiner l'existence du texte auctorial pour lui en substituer un autre, au lieu de faire apparaître la densité sémantique particulière du texte littéraire.

On pourrait, sur le même mode s'amuser à réécrire Madame Bovary en supposant que la mort de l'héroïne n'est pas un suicide mais un assassinat et s'offrir l'apparence de quelque vraisemblance. En effet, au sens figuré, le roman n'exempte pas l'entourage d'Emma d'une responsabilité dans sa mort. Flaubert souligne la lâcheté de ses anciens amants Rodolphe et Léon, la bassesse de Lheureux, le négociant prêteur, la complicité passive de Homais dans la boutique de qui elle prend l'arsenic, lequel Homais suggère qu'il s'agit d'une mort accidentelle, la farine pour un gâteau ayant été confondue avec de la mortaux-rats. La confusion entre interprétation et réécriture de la trame narrative serait pourtant contreproductive. C'est parce que, à un premier niveau de lecture, le suicide de l'héroïne est une donnée irréfutable dans l'espace de la diégèse, que l'interprétation peut prendre son

\footnotetext{
${ }^{3}$ Charles déclare, il est vrai, vouloir: "ressourcer en quelque sorte l'activité critique sur une activité de production, non en tant que la critique est créatrice (de sens), ce que l'on accordera volontiers par ailleurs, mais au sens où l'on débouche sur d'autres textes au premier degré " (Introduction à l'étude des textes, p. 43). La concession accordée à une critique créatrice de sens nous semble ouvrir une direction plus féconde que la création d'autres textes au premier degré, avatars de la théorie de l'énoncé fantôme.

${ }^{4}$ Nous avons tenté ce genre d'application aux fragments rescapés de La Défense de l'infini. Voir à ce sujet $L e$ lecteur et le livre fantôme, Essai sur La Défense de l'infini de Louis Aragon, Paris, Kimé, 2000.

${ }^{5}$ Nous ne partageons pas la tendance consistant, au nom de la théorie des textes possibles, à gommer la différence de statut entre texte virtuel et texte effectivement arrêté par l'auteur. Nous partageons à ce sujet les réserves de Nancy Murzilly exprimées dans "Logique et ontologie de la "case aveugle ": sur le statut du possible en littérature ", La lecture littéraire, $n^{\circ} 8$, " La case blanche », Paris, L'Improviste, 2006, p. 31-40. Elle précise notamment : "La recherche des possibles ne présuppose pas l'existence ontologique de virtualités non encore réalisées » (p. 37).
} 
essor sur le sens à lui attribuer : entre grandeur tragique d'une héroïne incomprise de ses contemporains et sanction de l'échec d'une vie par ailleurs par ailleurs traitée sur le mode du grotesque triste, invention flaubertienne.

II semble plus pertinent, donc, d'envisager création auctoriale et création lectorale comme activités socialisées complémentaires que de les confondre.

\section{Fondements d'une littérarité de la lecture}

Précisons pour conclure ce que recouvre à notre avis la notion de lecture littéraire. Elle implique, contrairement à certaines typologies (Goodman, 1968) qui réservent cet aspect à des arts comme la musique, de concevoir la littérature comme art à deux temps : onde de choc et répliques. Elle reposerait sur quatre aspects.

Respect de l'artefact auctorial, d'abord. Face aux objets incertains, se refusant à la lisibilité immédiate, elle procèderait au démontage de l'ambiguïté générique du texte à lire. En écho à la profusion d'une écriture soumise aux caprices de l'imaginaire, la lecture mise en mots peut faire émerger des structures interprétatives : au scénario narratif brouillé elle tend alors à substituer un scénario fantasmatique reconnu comme construction lectorale.

Cette reconstruction suppose, seconde condition, l'investissement affectif du texte d'auteur sur le mode de l'écoute flottante. Selon le titre d'une belle collection fondée par Jean Bellemin-Noël, on peut alors dire que «le texte rêve ", ou que le lecteur rêve à propos du texte lu, prolongeant l'aventure créatrice par une investigation touchant à une intersubjectivité, celle de l'auteur et la sienne.

Pour donner sa pleine efficacité à la lecture littéraire, il faut encore que le lecteur puisse déployer à son tour une mémoire intertextuelle susceptible d'entrer en résonance avec celle de l'auteur. Qu'il soit en mesure de percevoir l'allusion là où le texte n'exhibe pas sa connivence intertextuelle, le refoulement, là où la référence devient fragmentaire et masquée. On peut ainsi retrouver la trace de Sartre dans La Tante Julia et le scribouillard (Gladieu, 2006), à une époque où Vargas Llosa s'est détourné de son modèle de jeunesse et pas seulement dans La ville et les chiens, qui s'ouvrait sur une épigraphe empruntée au père de l'existentialisme.

Enfin, on ne saurait envisager de création lectorale ni de lecture littéraire au plein sens du terme sans concrétisation dans un texte de lecture qui substitue aux effets de sens virtuels entrevus dans une simple lecture «muette» une élaboration plus tangible et communicable. Ce texte de lecture, formation de compromis entre le texte d'auteur et la culture du lecteur, convertit le plaisir de lire en plaisir de dire la lecture, une approche intuitive d'effets imbriqués et superposés en un contre-texte plus rationalisé qui vaut moins pour lui seul que comme incitation à retourner au texte commenté. Délibérément, cette vision 
de la lecture se heurte à une égalisation générale de tous les énoncés, en tant que sources de richesse intellectuelle et de plaisir esthétique. 


\section{Bibliographie}

ARRIVE, Michel (1973). "Pour une théorie des poly-isotopiques ». Langages, 31.

CAmus, Albert (1964-1974), Lettre à Elsa Triolet. In : CEuvres Romanesques Croisées d'Aragon et d'Elsa Triolet. Paris : Robert Laffont, 42 volumes.

CHARLES, Michel (1995). Introduction à l'étude des textes. Paris : Le Seuil.

FAULKNER, William (1931, 1972). Sanctuaire. Paris : Gallimard, trad. R. N. Raimbault et Henri Delgove.

FUENTES, Carlos (1993, 1995, 2007). Las dos orillas, Les deux rives. Paris : Gallimard, trad. Céline Zins ; rééd., « folio bilingue ».

Eco, Umberto (1990, 1992). Les limites de l'interprétation. Paris : Grasset, trad. Myriem Bouhazer.

GervaIS, Bertrand $(1993,2006)$. A l'écoute de la lecture. Qc : VLB ; rééd. Notabene.

GLADIEU, Marie-Madeleine (2006). « Mario Vargas Llosa et Jean-Paul Sartre » : In Marie-Madeleine Gladieu, Alain Trouvé (dir.), "Parcours de la reconnaissance intertextuelle », Approches Interdisciplinaires de la Lecture, $\mathrm{n}^{\circ}{ }^{1}$. Reims : Presses Universitaires de Reims.

Goodman, Nelson (1968, 1990, 2005). Langages de l'art. Nîmes : Jacqueline Chambon, trad. Roger Pouivet ; rééd., Paris, Hachette.

LA LECTURE LITTERAIRE, $n^{\circ} 8$ (2006). Marc Escola, Sophie Rabau (dir.) . «La case blanche ». Paris : L'Improviste.

LA LECTURE LITTERAIRE, n`9 (2007). Alain Trouvé (dir.). « Lecture et psychanalyse ». Paris : L'Improviste.

MURZILlY, Nancy (2006). "Logique et ontologie de la 'case aveugle' : sur le statut du possible en littérature ». In : Marc Escola, Sophie Rabau (dir.), La lecture littéraire, $n^{\circ} 8$, « La case blanche », Paris, L'Improviste.

PICARD, Michel (1986). La Lecture comme jeu. Paris : Minuit.

RANCIERE, Jacques (2007). Politique de la littérature. Paris : Galilée.

ROBERT, Marthe (1972). Roman des origines et origines du roman. Paris : Gallimard.

RCHAEFFER, Jean-Marie (1999). Pourquoi la fiction ? Paris : Le Seuil.

RRIOLET, Elsa (1964-1974). CEuvres Romanesques Croisées d'Aragon et d'Elsa Triolet. Paris : Robert Laffont, 42 volumes.

Trouve, Alain (2000). Le lecteur et le livre fantôme, Essai sur La Défense de l'infini de Louis Aragon. Paris : Kimé.

Trouve, Alain (2007). "Sur les traces de l'intertexte latent ». In : Alain Trouvé (dir.). La Lecture littéraire, $n^{\circ} 9$, « Lecture et psychanalyse ». Paris : L'Improviste.

WinNicotT, DONALD W. $(1971,1975)$. Jeu et réalité - L'espace potentiel. Paris : Gallimard, trad. Claude Monot et J.-B. Pontalis. 